Submitted to Nano Letters, Jan 2004

\title{
Crystalline Boron Nanoribbons: Synthesis and Characterization
}

\section{Supporting Information}

Optical properties of boron and boron-rich materials are markedly affected by impurities and structural differences. Extensive research has been done on correlating optical properties to crystal structures, and the main results can be found in a recent review article. ${ }^{1}$ However, there is little data

for $\alpha$-tetragonal boron. For example, infrared (IR) spectra were only reported by Becher, ${ }^{2}$ Weiheit $^{1}$ and Sugaya, ${ }^{3}$ respectively $\square$ and these authors disagree with each other about which peaks are present or absent. Here, we report IR and Raman spectra collected from the puffy ball material; we note that we have assigned the components of these ribbons as $\alpha$-tetragonal boron nanoribbons but only by TEM due to the limited quantity of material. Clearly, with respect to $\alpha$-tetragonal boron, future work will be needed on samples that can be proven to be pure, or for which any other components can themselves be uniquely assigned as to IR and Raman.

The IR spectra were obtained with a NEXUS 870 Fourier Transform Spectrometer (Thermo Nicolet Corp.). Figure 1 shows the IR absorption spectrum. We tentatively assign the major peaks as (i) the peaks at $698,800,849,984,1070,2843$ and $2917 \mathrm{~cm}^{-1}$ are due to boron lattice vibrations. ${ }^{2,4}$ (ii) The bands at $1397,1621,1965 \mathrm{~cm}^{-1}$ are due to vibrations involving the $\mathrm{B}-\mathrm{O}$ bond and other bonds attached to the $\mathrm{B}$ or the $\mathrm{O}$ of the B-O bond., (iii) The bands between $1000-1200 \mathrm{~cm}^{-1}$ are assigned as due to B-C vibration. (iiii) The band at $3432 \mathrm{~cm}^{-1}$ is assigned to an $\mathrm{O}-\mathrm{H}$ group which has been previously attributed to adsorbed water on boron oxide ${ }^{5,6}$. 


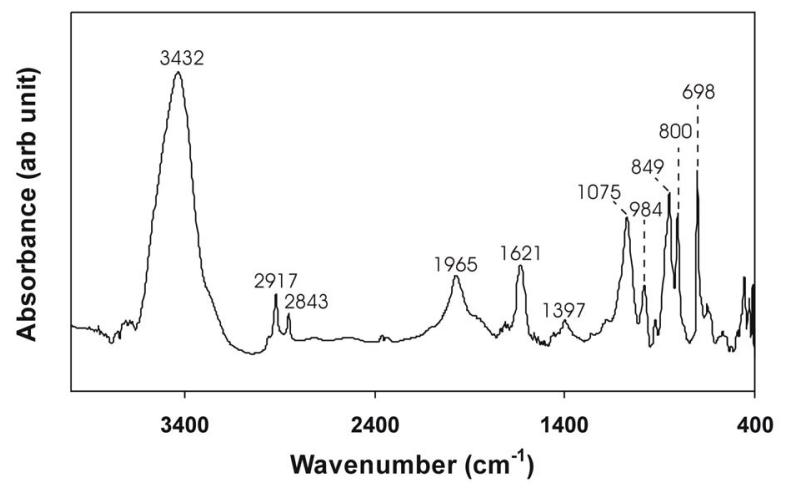

Figure 1. IR absorption spectrum of $\alpha$-tetragonal boron nanoribbons.

The Raman spectra were collected with a Renishaw Raman microscope $(514.5 \mathrm{~nm}$ Argon ion laser excitation). Figure 2 shows the Raman spectrum. The main peaks are at 366, 490, 642, 705, $746,795,910$, and $1100 \mathrm{~cm}^{-1}$. By analogy to the assignments of the Raman spectrum for each of $\alpha$ and $\beta$-rhombohedral boron, ${ }^{1}$ we assign the features above $600 \mathrm{~cm}^{-1}$ to vibrational modes of the $\mathrm{B}$ icosahedra in $\alpha$-tetragonal boron. Please note that the peaks we observed do not coincide exactly with those of $\alpha$ - and $\beta$-rhombohedral boron Raman peaks. ${ }^{1}$ We suggest that calculation of the Raman and IR active modes of $\alpha$-tetragonal boron is relevant to assist assignment of the experimental spectra.

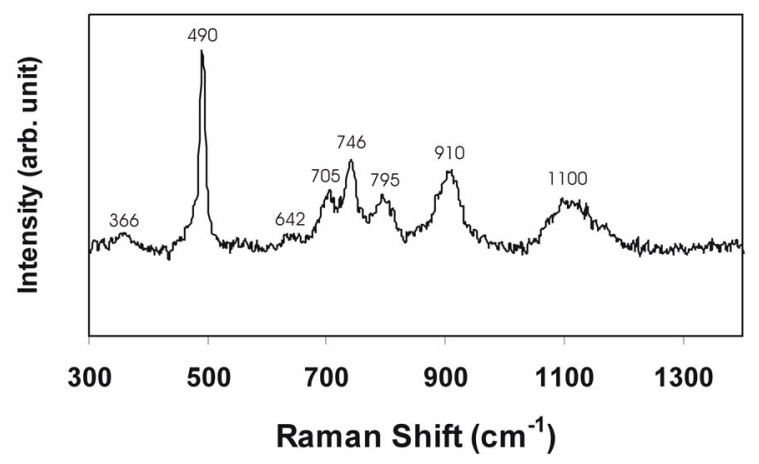

Figure 2. Raman spectrum of $\alpha$-tetragonal boron nanoribbons. 
1. Werheit, H. Prog. Crystal Growth and Charact. 1988, 16, 179.

2. Becher, H. J. Z. Anorg. Allg. Chem. 1963, 321, 216.

3. Sugaya, T.; Takeuchi, Y.; Watanabe, O. J. Phys. D: Appl. Phys. 1973, 6, L98.

4. Yu, S.; Ji, Y.; Li, T.; Han, M.; Wang, G.; Yuan, X.; Li, Z.; Chen, P. Solid State Commun. 2000, $115,307$.

5. $\quad$ Parsons, J. L.; Milberg, M. E. J. Amer. Ceram. Soc. 1960, 43, 326.

6. Godet, C.; Schmirgeld, L.; Zuppiroli, L.; Sardin, G.; Gujrathi, S.; Oxorn, K. J. Mat. Sci. 1991, 26,6408 .

7. Blum, N. A.; Feldman, C.; Satkiewicz, F. G. Phys. Stat. Sol. (a) 1977, 41, 481. 